\title{
Enhancement on antioxidant and antibacterial activities of Brightwell blueberry by extraction and purification
}

\author{
Haonan Liu ${ }^{1,2}$, Han Wu ${ }^{2}$, Ying Wang ${ }^{2}$, Fan Wang ${ }^{2}$, Xiaoli Liu ${ }^{1,2}$ and Jianzhong Zhou ${ }^{1,2^{*}}$
}

\begin{abstract}
A blueberry anthocyanin extract was obtained from Brightwell blueberry fruits cultivated in eastern China and the extraction and purification conditions were optimized. The components of the anthocyanin extract were identified using ultra-performance liquid chromatography-electrospray ionization interface-mass spectrometer. The antioxidant and antibacterial activities of the blueberry fruit supernatant (BFS), blueberry anthocyanin crude extract (BCE), and blueberry anthocyanin rich extract (BRE) were evaluated. The extraction yield was $1.79 \pm 0.0014 \mathrm{mg} / \mathrm{g}$ under the following optimal conditions: 1:20 solid-to-liquid ratio $(\mathrm{V} / \mathrm{W}), 24 \mathrm{~h}, 34^{\circ} \mathrm{C}$, and $90 \%$ ethanol containing $0.21 \%(\mathrm{~V} / \mathrm{V})$ hydrochloric acid. With regard to purification, anthocyanin purity increased 19.1-fold. Nine fractions were identified as the glycosides of delphinidin, cyanidin, petunidin, and malvidin. The biological activities of the blueberry anthocyanin extract were improved through extraction and purification. Compared with BFS and BCE, BRE had a higher DPPH radical scavenging activity $\left(E C_{50}=0.51 \mathrm{mg} / \mathrm{mL}\right)$, ABTS antioxidant capacity $\left(\mathrm{EC}_{50}=0.32 \mathrm{mg} / \mathrm{mL}\right)$, and oxygen radical absorbance capacity $(0.43 \mathrm{mmol}$ Trolox/g). Furthermore, BRE $(2 \mathrm{mg} / \mathrm{mL})$ showed a maximum of $84.64 \pm 0.35 \%$ reduction in the biofilm biomass of Listeria monocytogenes and the inhibition zone given by BRE against Escherichia coli was $16.04 \pm 0.38 \mathrm{~mm}$. BRE showed the highest antioxidant capacities and obvious antibacterial effects against foodrelated microorganisms than the other samples. Therefore, BRE can be used as a natural antioxidant and antibacterial agent and has potential health advantages and food industry applications.
\end{abstract}

Keywords: Blueberry anthocyanin, Extraction and purification, Anthocyanin characterization, Antioxidant, Antibacterial

\section{Introduction}

Chemical preservatives are often added to improve microbiological food safety. Consumers, on the other hand, are becoming increasingly concerned about the possible dangers of chemical preservatives and want meals that are free of them. Furthermore, they prefer natural antioxidants produced from natural sources since they are safer and have less adverse effects than synthetic antioxidants. As a result, the demand for novel natural

\footnotetext{
*Correspondence: 1939650782@qq.com

'School of Food and Biological Engineering, Jiangsu University, 301

Xuefu Road, Zhenjiang, Jiangsu 212013, P.R. China

Full list of author information is available at the end of the article
}

food additives that can act as antibacterial and antioxidant agents is growing. Hence, plant materials with high antioxidant and antibacterial properties are crucial.

Blueberries (Vaccinium spp.) have a wide range of health advantages and nutritional components [1]. Blueberries are known for their excellent antioxidant properties, which are attributed to their high total anthocyanin content [2]. Anthocyanin varieties contain different levels of glycosides, providing colors to flowers, fruits, and other organs of plants [3]. Anthocyanin also has great potential benefits to human health due to its strong oxidation resistance for enhancing immunity capacity and balancing weight $[1,4-6]$. Sun investigated the inhibition effects of anthocyanins found in wild blueberries on the 
growth of Listeria monocytogenes, Staphylococcus aureus, Salmonella enteritidis, and Vibrio parahaemolyticus [7]. Industrially-used methods for extracting anthocyanins from natural sources are commonly based on conventional solvent extraction [8]. Owing to the unique qualities of bioactive compounds, such as high adsorption ability and convenient desorption, absorption separation with macroporous resins is an effective technique for isolating anthocyanins from plant crude extracts [9].

As previously reported, the composition of anthocyanins in blueberry varieties grown at different regions, years, and harvest times greatly varies. Although anthocyanin extraction and purification have been widely explored, there is currently a scarcity of research focusing on the constitution of anthocyanins and their contributions to the health benefits of the blueberry cultivar Brightwell. Therefore, the extraction of anthocyanins from Brightwell blueberry was optimized using response surface methodology in this study, and the macroporous resin AB-8 was used in purifying the crude extract. The blueberry anthocyanins were then characterized through UPLC-ESI-MS/MS. Notably, the only solvent used was ethanol, guiding the safe and efficient utilization of foodgrade blueberry anthocyanins. Moreover, the blueberry anthocyanin rich extract (BRE) showed great antioxidant and antibacterial capacities, and it can be used as a natural antioxidant and antibacterial agent, showing potential health advantages and food industry applications.

\section{Materials and methods}

\section{Materials and chemicals}

The blueberry cultivar "Brightwell" was collected and stored at $-18^{\circ} \mathrm{C}$ in Baima Planting Base (Nanjing, China) in July 2020. HPLC-grade acetonitrile, methanol, and formic acid were purchased from Merck Chemicals Co., Ltd. (Darmstadt, Germany). 2,2-diphenyl-1-picrylhydrazyl (DPPH), 2,2-azinobis (3-ethylbenzothiazoline-6-sulfonic acid) diammonium salt (ABTS), 2,2'-Azo-bis(2-amidinopropane) dihydrochloride (AAPH), 6-hydroxy-2,5,8tetramethylchroman-2-carboxylic acid (Trolox), and fluorescein disodium salt were purchased from SigmaAldrich (St. Louis, MO, USA). All other solvents and chemicals were of analytical grade obtained from Sinopharm Chemical Reagent (Shanghai, China).

\section{Blueberry anthocyanin extraction and single-factor analysis}

Fresh berries were smashed and centrifuged for $20 \mathrm{~min}$ at $5000 \mathrm{rpm}$. The obtained blueberry fruit supernatant (BFS) was freeze-dried for further study. The blueberry anthocyanin was extracted from smashed berries by using a mixture of ethanol, water, and $\mathrm{HCl}$ at $150 \mathrm{rpm}$. The crude extract was centrifuged for $15 \mathrm{~min}$ at
$5000 \mathrm{rpm}$, and the supernatant was diluted in $\mathrm{HCl}$ ethanol solution to $50 \mathrm{~mL}$. The variables that tested included: $\mathrm{HCl}$ concentration $(0.05,0.10,0.25,0.30,0.75$, and $1 \%)$, ethanol concentration (50-100\%), solid to liquid ratio $(1: 3,1: 5,1: 10,1: 15,1: 20$, and $1: 25 \mathrm{v} / \mathrm{w})$, extraction temperature $\left(20-60{ }^{\circ} \mathrm{C}\right)$ and extraction time $(1,3,6,12$, and $18 \mathrm{~h}$ ) were initially applied as single factors.

\section{Optimization of the Box-Behnken response surface}

The conditions for blueberry anthocyanin extraction were optimized through response surface methodology (RSM). A Box Behnken design was used in investigating the effects of independent variables, namely, ethanol concentration (A), $\mathrm{HCl}$ dose (B), extraction time (C), extraction temperature $(D)$, and solid-to-liquid ratio $(E)$ on the yield of blueberry anthocyanin extracts. The experimental design is shown in Table 1 in coded and actual levels of variables. The blueberry anthocyanin crude extract (BCE) was obtained by using the optimized extract conditions. BCE was further freeze-dried for further study.

\section{Determination of the yield of total anthocyanin}

Anthocyanin pigment concentrations in the crude extracts were determined using the spectrophotometric $\mathrm{pH}$ differential approach [10]. Absorbances were determined at 520 and $700 \mathrm{~nm}$ with a UV-P5 visible spectrophotometer (Shanghai Mapada Instrument Co., Ltd., Shanghai, China). The extraction yield of anthocyanin was calculated as follows:

$$
\mathrm{C}=\frac{\Delta \mathrm{A} \times \mathrm{M} \times \mathrm{f} \times \mathrm{V}}{\varepsilon \times \mathrm{m} \times \mathrm{L}}
$$

where $C$ is the extraction yield of anthocyanin $(\mathrm{mg} / \mathrm{g})$, $\Delta \mathrm{A}=\left(\mathrm{A}_{520 \mathrm{~nm}}-\mathrm{A}_{700 \mathrm{~nm}}\right) \mathrm{pH}_{1.0}-\left(\mathrm{A}_{520 \mathrm{~nm}}-\mathrm{A}_{700 \mathrm{~nm}}\right) \mathrm{PH}_{4.5}$ $M$ is the molecular weight of cyanidin-3-glucoside $(449.2 \mathrm{~g} / \mathrm{mol}), \mathrm{f}$ is the dilution factor of extract, $\mathrm{V}$ is the volume of extract $(\mathrm{mL}), \varepsilon$ is the molar extinction coefficient of cyanidin-3- glucoside $(26,900 \mathrm{~L} / \mathrm{mol} \cdot \mathrm{cm}), \mathrm{L}$ is the cuvette length $(\mathrm{cm})$, and $\mathrm{m}$ is the weight of the sample (g).

Table 1 Levels and codes of factors for Box-Behnken experimental design

\begin{tabular}{lllll}
\hline Factor & \multicolumn{4}{l}{ Coding level } \\
\cline { 3 - 5 } & & $-\mathbf{1}$ & $\mathbf{0}$ & $\mathbf{1}$ \\
\hline Ethanol concentration $(\%)$ & $\mathrm{A}$ & 50 & 70 & 90 \\
$\mathrm{HCl}$ dosage $(\%)$ & $\mathrm{B}$ & 0.05 & 0.40 & 0.75 \\
Extraction time $(\mathrm{h})$ & $\mathrm{C}$ & 2 & 14 & 26 \\
Extraction temperature $\left({ }^{\circ} \mathrm{C}\right)$ & $\mathrm{D}$ & 25 & 50 & 75 \\
Solid-to-liquid ratio $(\mathrm{g} / \mathrm{mL})$ & $\mathrm{E}$ & $1: 5$ & $1: 15$ & $1: 25$ \\
\hline
\end{tabular}


Static adsorption and desorption properties of the resins The preparation of the resins was according to the method of Gao [1]. In pretreatment of macroporous resin, AB-8 macroporous resin powder was soaked in 95\% alcohol aqueous until it fully swelled (approximately $24 \mathrm{~h}$ ), and then cleaned by leaching pure alcohol to neatness.

The activated adsorbent (5 g dry weight) was added to $50 \mathrm{~mL}$ of sample solution in a sealed flask at approximately $25{ }^{\circ} \mathrm{C}$ in an orbital shaker at $150 \mathrm{rpm}$ for $24 \mathrm{~h}$. After adsorption, the concentration of anthocyanin in the filtrate was determined. The adsorption capacity and rate of anthocyanins in macroporous resin AB-8 were determined using the following formula:

$$
\begin{aligned}
& \mathrm{Q}=\frac{\left(\mathrm{C}_{0}-C_{r}\right) \times v}{\mathrm{~m}} \\
& \mathrm{~A}=\frac{\mathrm{C}_{0}-\mathrm{C}_{\mathrm{r}}}{\mathrm{C}_{0}} \times 100 \%
\end{aligned}
$$

where $\mathrm{Q}$ is the adsorption capacity $(\mathrm{mg} / \mathrm{g}), \mathrm{C}_{0}$ is the initial concentration of anthocyanin $(\mathrm{mg} / \mathrm{mL}), \mathrm{C}_{\mathrm{r}}$ is the equilibrium concentration of anthocyanin $(\mathrm{mg} / \mathrm{mL}), \mathrm{v}$ is the volume of adsorption solution $(\mathrm{mL}), \mathrm{m}$ is the mass of $\operatorname{resin}(\mathrm{g})$, and $\mathrm{A}$ is the adsorption rate (\%).

The macroporous resins that absorbed anthocyanin were rinsed three times with deionized water after adsorption equilibrium was achieved. Furthermore, the macroporous resins were desorbed with $50 \mathrm{~mL}$ of $100 \%$ ethanol solution in a sealed flask at approximately $25{ }^{\circ} \mathrm{C}$ in an orbital shaker at $150 \mathrm{rpm}$ for $24 \mathrm{~h}$. The anthocyanin desorption rate of macroporous resin AB- 8 was estimated as follows:

$$
\mathrm{D}=\frac{\mathrm{v} \times \mathrm{C}}{\mathrm{m} \times \mathrm{Q}} \times 100 \%
$$

where $\mathrm{Q}$ is the adsorption capacity ( $\mathrm{mg} / \mathrm{g}$ ), $\mathrm{C}$ is the content of anthocyanins in the solution after desorption ( $\mathrm{mg}$ $/ \mathrm{mL}), \mathrm{v}$ is the volume of desorption solution $(\mathrm{mL}), \mathrm{m}$ is the mass of resin $(\mathrm{g})$, and $\mathrm{D}$ is the desorption rate (\%).

\section{Blueberry anthocyanin purification and single-factor analysis}

BCE was loaded into an AB-8 macroporous resin column and eluted in a similar way to the method of Gao [1]. Activated macroporous resins were employed under different adsorption conditions. A single-factor experiment was used in assessing the influence of the factor in terms of anthocyanin concentration $(0.5,1,2,3,4,5$, 6 , and $7 \mathrm{mg} / \mathrm{mL}$ ) on $\mathrm{AB}-8$ macroporous resin adsorption rate and two variables in terms of elution flow rate and ethanol concentration on AB-8 macroporous resin desorption rate. Equations (3) and (4) were used in calculating the adsorption and desorption rates of $\mathrm{AB}-8$ macroporous resin, respectively. BCE was purified using AB-8 resin and freeze-dried. The obtained blueberry anthocyanin rich extract (BRE) was used in further study.

\section{Identification of anthocyanin by UPLC-PDA-ESI-MS}

The sample was examined using a the UPLC-MS system (G2-XS QTof, Waters). With a $0.35 \mathrm{~mL} / \mathrm{min}$ flow rate, $2 \mu \mathrm{L}$ of the sample was injected into the UPLC column (2.1 mmX100 mm ACQUITY UPLC BEH C18 column containing $1.7 \mu \mathrm{m}$ particles). Buffer A contained water and $0.1 \%$ formic acid, while buffer B consisted of $0.1 \%$ formic acid in acetonitrile. The gradient was $5 \%$ buffer B for $0.5 \mathrm{~min}, 5-40 \%$ buffer B for over $20 \mathrm{~min}, 40-95 \%$ buffer B for over $2 \mathrm{~min}$.

At a mass range of $50-1200 \mathrm{~m} / \mathrm{z}$, mass spectrometry was performed, and an electrospray source was used in positive ion mode with MSe continuum acquisition mode. Recalibration with leucine-enkephalin $(\mathrm{m} / \mathrm{z}$ 556.2771 for positive ion mode and 554.2615 for negative ion mode) activated the lock mass option. The capillary voltage was $2.0 \mathrm{kV}$, the collision energy was $10-40 \mathrm{eV}$, the source temperature was $120{ }^{\circ} \mathrm{C}$, and the desolvation gas temperature was $400{ }^{\circ} \mathrm{C}$, according to the ionisation parameters. Masslynx 4.1 and UNIFI were used to collect and process the data (Waters Company).

\section{Measurement of antioxidant activities}

Freeze-dried BRE, BCE, and BFS dissolved in deionized water at the different concentrations were used in the antioxidant assays. Antioxidant activity of samples was determined using several previously reported different antioxidant assays such as DPPH radical scavenging assay (DPPH), ABTS radical cation scavenging assay (ABTS), oxygen radical absorbance capacity (ORAC) $[11,12]$.

\section{Antimicrobial activity Bacterial strains}

The test organism Listeria monocytogenes $(21,532)$ was provided by China Center of Industrial Culture (CICC). The test organism Salmonella enterica (ATCC 51,812) and Escherichia coli (ATCC 25,922) were obtained from Nanjing Institute of Supervision and Testing on Product Quality (Jiangsu, China). Bacteria were cultivated at $37^{\circ} \mathrm{C}$ for $24 \mathrm{~h}$. Listeria monocytogenes were used to determine biofilm formation. Salmonella enterica, Escherichia coli, and Listeria monocytogenes were used to determine the potential antibacterial activities of samples. 


\section{Anti-biofilm activity}

The effects of the samples on biofilm formation were evaluated by the approach of Zhang [13]. Briefly, 1\% of tested bacteria $\left(0.4 \mathrm{OD}_{600 \mathrm{~nm}}\right)$ with different concentrations of samples $(0.1,0.5$, and $2 \mathrm{mg} / \mathrm{mL})$ were inoculated to $\mathrm{LB}$ medium. $1 \mathrm{~mL}$ of the mixture above was added to each well of 48-well plates. The plates were cultivated at $37^{\circ} \mathrm{C}$ for $24 \mathrm{~h}$. When quantifying the biofilm, plates were washed by deionized water, stabilized by methyl alcohol, and stained with $0.1 \%$ crystal violet. The biofilms were washed three times with deionized water for removal excess dye. $1 \mathrm{~mL}$ of $95 \%$ ethanol was used to dissolve the dye adhered to the biofilm. The biomass of the biofilm was represented as the OD measured at $595 \mathrm{~nm}$.

\section{Determination of the diameter of inhibition zone (DIZ)}

The effects of the samples on antibacterial properties were determined using the disk diffusion assay. The diameter of inhibition zone (DIZ) was determined using the method of Gan [14]. Three bacteria, including, Salmonella enterica, Escherichia coli, and Listeria monocytogenes were used to test the antibacterial activity of samples. In brief, the strains were incubated for $24 \mathrm{~h}$ in LB medium at optimal temperature. $1 \%$ of the tested bacterial suspension $\left(0.8 \mathrm{OD}_{600 \mathrm{~nm}}\right)$ was evenly spread on the LB agar plate. $100 \mu \mathrm{L}$ of each sample $(0.5 \mathrm{mg} / \mathrm{mL})$ was transferred into an Oxford cup, which was lightly set on an agar plate in advance. Sterile water was applied as the positive control. Afterwards, the agar plates were incubated at $37^{\circ} \mathrm{C}$ for $24 \mathrm{~h}$. The antibacterial activities of the samples were determined by measuring the inhibition zones with a ruler. The test was repeated three times and the results were measured and expressed in millimeters (mm). "No inhibition zone (NIZ)" samples had a DIZ of less than $8.0 \mathrm{~mm}$.

\section{Statistical analysis}

All the data were expressed as means \pm standard deviation (SD) from triplicate experiments. Analysis of variance (ANOVA) was used to analyze the data, followed by Duncan's Multiple Range Test (SPSS version 20.0, SPSS Inc., Chicago, IL, USA), and the significance level was $P<0.05$.

\section{Results and discussion}

\section{Single-factor experimental analysis of blueberry anthocyanin extraction}

The extraction process should not be overly complicated, dangerous, time consuming and expensive [15]. Because of its low toxicity and good extraction capacity, ethanol is commonly applied in the food industry. The permeability of a solvent increases with its concentration. This feature is the conducive to the dissolution of water-soluble substances. Increase in ethanol concentration inhibits the dissolution of sugars, pectins, and other highly polar water-soluble pigments, and the yield of anthocyanins is enhanced. Figure 1a showed the influence of ethanol concentration on blueberry anthocyanin extraction yield. When the ethanol concentration was increased from 50 to $70 \%$, the anthocyanin production was positively related to the ethanol concentration. At ethanol concentration of $70 \%$, the anthocyanin output increased considerably and peaked at $1.58 \pm 0.0015 \mathrm{mg} / \mathrm{g}(P<0.001)$. Kang extracted anthocyanins with $70 \%$ ethanol and obtained good results [15].

In an acidic environment, the majority of anthocyanins are stable [16]. Complex bonds between anthocyanins and metal ions, the ionic bonds between polysaccharides and proteins, and the hydrogen bonds can be broken simultaneously. Thus, this environment promotes the dissolution of anthocyanins and increases its yield. However, the addition of excessive strong acids partially hydrolysis glycosidic bonds and acyl groups of anthocyanins, thus affecting the anthocyanin extraction yield [17]. Figure $1 \mathrm{~b}$ presented the influence of $\mathrm{HCl}$ concentration on the extraction yield of anthocyanin. When the dosage of $\mathrm{HCl}$ was $0.5 \%(\mathrm{v} / \mathrm{v})$, the greatest extraction yield of anthocyanin was $1.89 \pm 0.11 \mathrm{mg} / \mathrm{g}$.

The length of time between extractions might affect the yield of anthocyanin. Extraction increases with time before the desired components inside and outside plant cells reach equilibrium [18]. It was seen from Fig. 1c that approximately $1.22 \pm 0.0045 \mathrm{mg} / \mathrm{g}$ of anthocyanin was extracted from blueberry during the first $12 \mathrm{~h}$. Therefore, the $12 \mathrm{~h}$ was considered sufficient for extracting the anthocyanin from blueberry.

In addition, the extraction of heat-sensitive chemicals is influenced by temperature. Molecular thermal mobility and solvent diffusion, penetration, and dissolution rates increase with temperature. Molecules easily transfer to solvents, and the dissolution of impurities results in the disintegration of some labile thermal components, such as anthocyanins [19]. The results indicated that the extraction yield of anthocyanin increased initially and decreased with increasing temperature (Fig. 1d). Compared with other temperatures, $50{ }^{\circ} \mathrm{C}$ was the turning point for extracting anthocyanin and increased extraction efficiency $(P<0.05)$. Hence, the optimal temperature for extracting and separating blueberry anthocyanin was $50{ }^{\circ} \mathrm{C}$.

Another essential aspect of the extraction process is the solid-to-liquid ratio. A larger solvent volume dissolves polyphenol components effectively, leading to a high extraction yield. Anthocyanins are efficiently dissolved during extraction at a high solid-to-liquid ratio, and thus extraction yield increases. However, an extremely high 

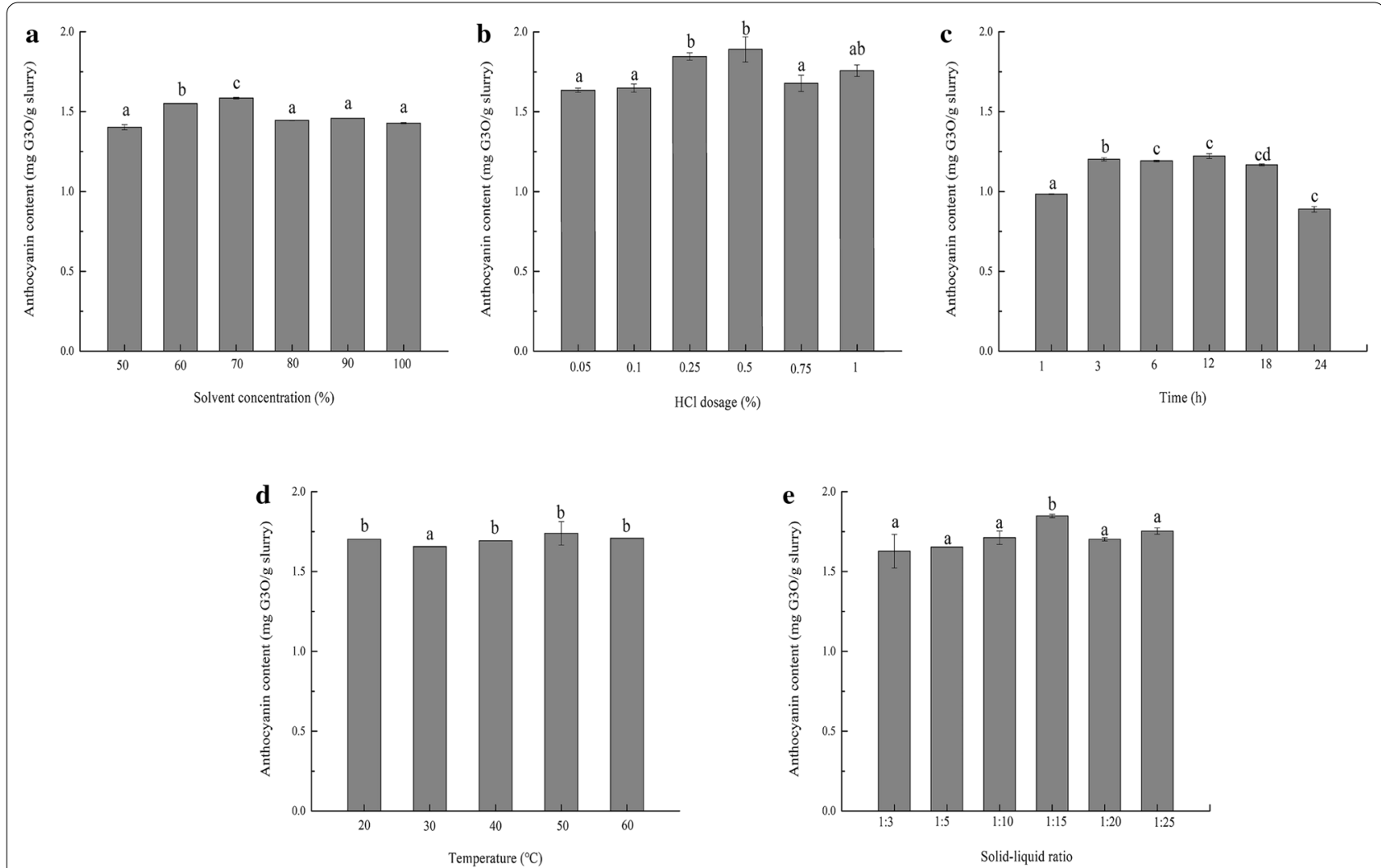

Fig. 1 Single-factor analysis of anthocyanin extraction from blueberry. The effects of different extraction parameters: $\mathbf{a}$ ethanol concentration, $\mathbf{b}$ $\mathrm{HCl}$ concentration, $\mathbf{c}$ extraction time, $\mathbf{d}$ extraction temperature, and e solid-to-liquid ratio on the anthocyanin extraction yields

solid-to-liquid ratio may cause the dissolution of impurities and then reduce the solubility of anthocyanins. As shown in Fig. 2e, the anthocyanin yield increased when the solid-to-liquid mass ratio increased from $1: 3 \mathrm{v} / \mathrm{w}$ to $1: 15 \mathrm{v} / \mathrm{w}$. When the mass ratio was $1: 15 \mathrm{v} / \mathrm{w}$, the extraction yield of anthocyanin reached its maximum.

According to the single factor experiment, the ethanol concentration $(50 \%, 70 \%$, and $90 \%)$, hydrochloric acid addition $(0.05 \%, 0.40 \%$, and $0.75 \%)$, extraction time (2, 14 , and $26 \mathrm{~h}$ ), extraction temperature $\left(25^{\circ} \mathrm{C}, 50^{\circ} \mathrm{C}, 75^{\circ} \mathrm{C}\right)$ and solid-liquid ratio $(1: 5,1: 15,1: 25 \mathrm{v} / \mathrm{w})$ were selected as the parameters for the subsequent response surface optimization.

\section{Optimization of blueberry anthocyanin extraction with Box-Behnken design (BBD)}

The RSM approach was used in optimizing the anthocyanin yield from blueberry. The coded and actual levels of five variables presented in Table 1 . The response variable (yield) and variables were related by the following second-order polynomial equation: $\mathrm{Y}=1.29+$ $0.096 \mathrm{~A}-0.16 \mathrm{~B}-0.026 \mathrm{C}-0.030 \mathrm{D}+0.10 \mathrm{E}+0.00337$ $2 \mathrm{AB}+0.058 \mathrm{AC}+0.27 \mathrm{AD}+0.084 \mathrm{AE}-0.16 \mathrm{BC}-0.22$
$\mathrm{BD}-0.19 \mathrm{BE}-0.23 \mathrm{CD}+0.052 \mathrm{CE}+0.15 \mathrm{DE}+0.1 \mathrm{~A}^{2}$ $-0.14 \mathrm{~B}^{2}+0.043 \mathrm{C}^{2}-0.20 \mathrm{D}^{2}-0.096 \mathrm{E}^{2}$, adopting multiple regression analysis on the experimental data.

This model had a $P$ value of 0.0005 in the analysis of variance of the multiple regression model of response surface design outcomes, indicating that it was significant. The $R^{2}$ coefficient, $R^{2} \mathrm{~A}_{\mathrm{dj}}$ coefficient, and $F$ and $P$ values of lack of fit showed that the model can effectively increase anthocyanin extraction yield. Interactions among ethanol concentration, $\mathrm{HCl}$ concentration, and extract time between extract temperatures significantly influenced the yield of anthocyanin $(P<0.05)$.

Figure 2 showed the response surface analysis results for blueberry anthocyanin extraction. The maximum expected yield of anthocyanin $(1.87 \mathrm{mg} / \mathrm{g})$ was obtained under the following conditions: ethanol concentration of $90 \%, \mathrm{HCl}$ concentration of $0.21 \%$, temperature of $34.57{ }^{\circ} \mathrm{C}$ for $24.48 \mathrm{~h}$, and solid-to-liquid ratio of $1: 20$ $\mathrm{v} / \mathrm{w}$. An actual yield of $1.79 \pm 0.0011 \mathrm{mg} / \mathrm{g}$ was obtained under ideal conditions. According to the coincidence between the results above and our previous prediction, these conditions were applied for further study. 

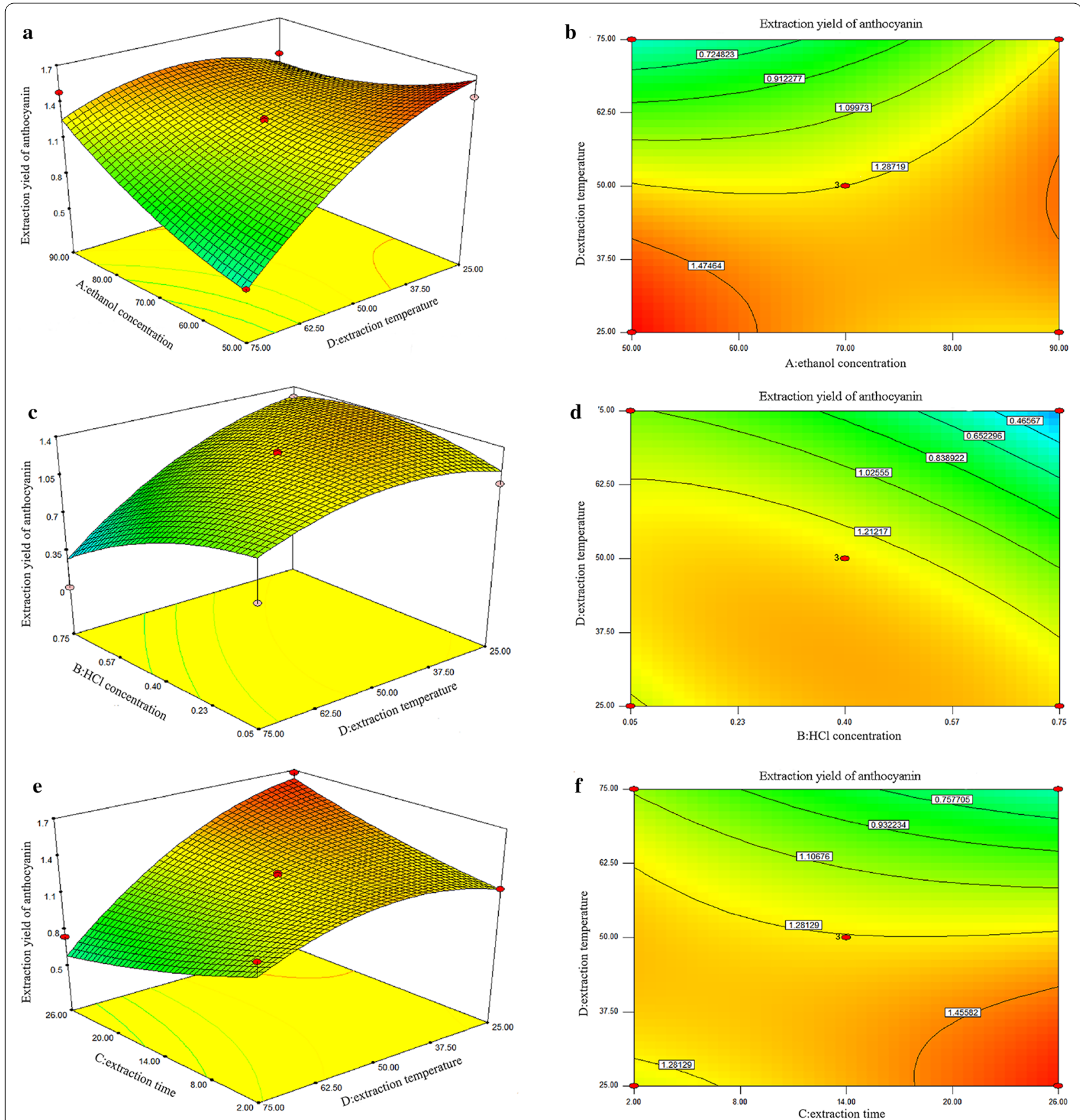

Fig. 2 Response surface analysis of anthocyanin extraction from blueberry. The interactive effect of ethanol concentration and extraction temperature on anthocyanin yield: a response surface plot and $\mathbf{b}$ contour plot. The interactive effect of $\mathrm{HCl}$ concentration and extraction temperature on anthocyanin yield: $\mathbf{c}$ response surface plot and $\mathbf{d}$ contour plot. The interactive effect of extraction time and extraction temperature on anthocyanin yield: e response surface plot and $\mathbf{f}$ contour plot

\section{Optimization of purification conditions of blueberry anthocyanin}

Figures $3 \mathrm{a}$ and $\mathrm{b}$ illustrated the kinetics curves for the adsorption and desorption of anthocyanins on $\mathrm{AB}-8$ resins. The adsorption ratio grew significantly for the first
$1.5 \mathrm{~h}$, then gradually increased in the next $1.5 \mathrm{~h}$ before reaching the equilibrium at approximately $17 \mathrm{~h}$. The equilibrium time for AB- 8 resin during the desorption process was $1.5 \mathrm{~h}$, at which the desperation ratio reached the highest at $99.99 \%$. Thus, in the following experiments, 

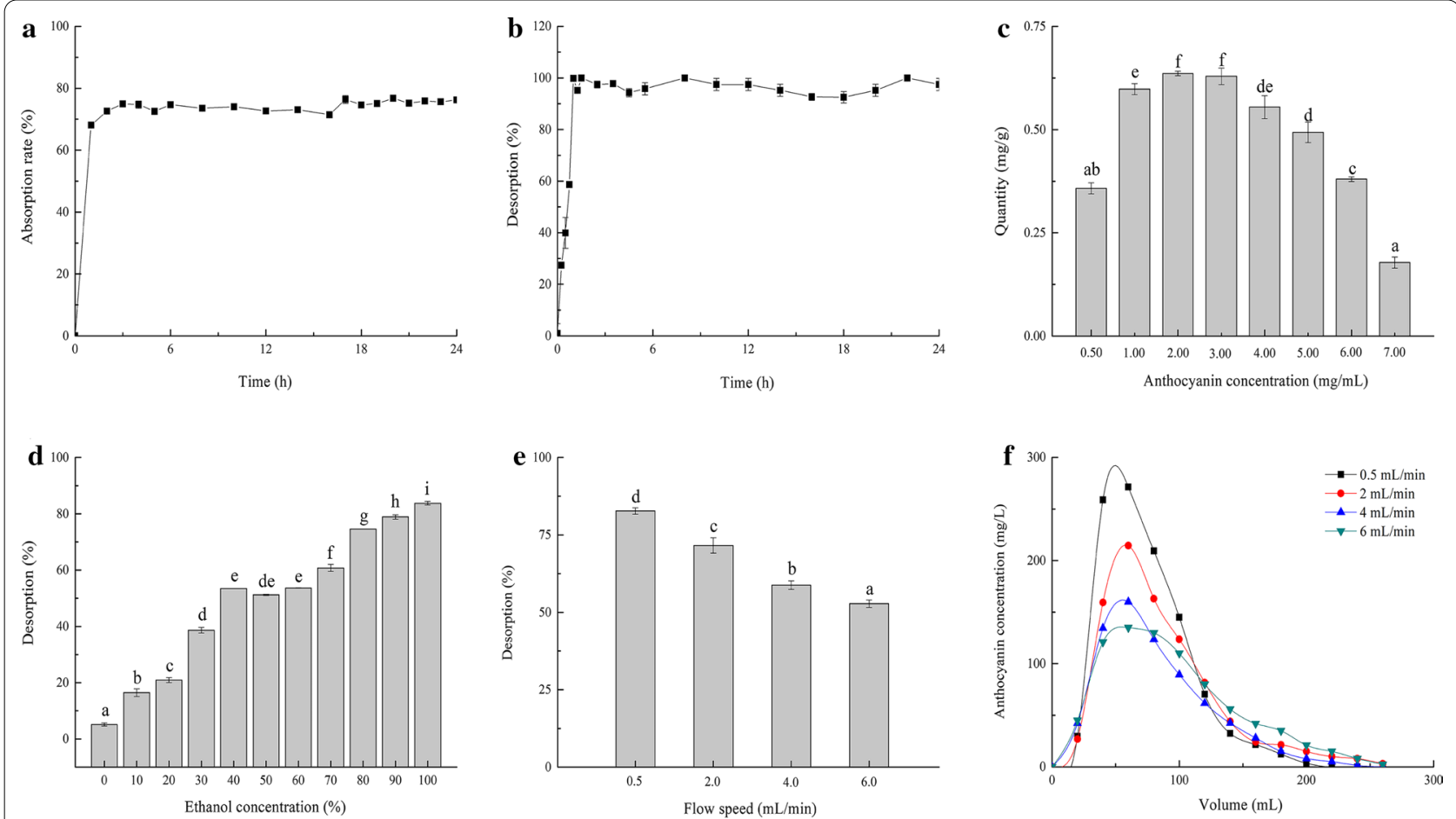

Fig. 3 Adsorption and desorption kinetics for anthocyanins on resins, and dynamic breakthrough and diffusion curves for anthocyanins on AB-8 resin. $\mathbf{a}$ the adsorption ratio and $\mathbf{b}$ desorption ratio of anthocyanins on AB-8resin; $\mathbf{c}$ dynamic absorption curves of anthocyanin on $A B-8$ macroporous resin at different anthocyanin concentrations; $\mathbf{d}$ dynamic desorption curve for anthocyanins on AB-8 resin at different ethanol concentrations; e dynamic desorption ratio for anthocyanins on AB-8 resin at different flow speed; $\mathbf{f}$ dynamic desorption curve for anthocyanins on AB-8 resin at different flow speed

the adsorption time of $17 \mathrm{~h}$ and desorption time of $2 \mathrm{~h}$ were chosen.

Figure 3c depicted the influence of anthocyanin content on the adsorption capacity of AB-8 macroporous resin. As shown in Fig. 3c $2 \mathrm{mg} / \mathrm{mL}$, anthocyanins exhibited the highest adsorption. Anthocyanin content exceeding $3.0 \mathrm{mg} / \mathrm{mL}$ negatively affected the dynamic absorption rates of anthocyanins on the AB-8 resin. High concentrations anthocyanins rich in impurities inhibited the absorption of anthocyanins in the AB- 8 macroporous resin column. Impurities considerably affected the flow and diffusion rates of anthocyanin on the macroporous resins. Furthermore, the high anthocyanin concentrations caused flocculation and precipitation, resulting in pollution and macroporous resin obstruction [20]. Low anthocyanin content increased resin use and decreased impurity adsorption. Hence it can be inferred that $2.0 \mathrm{mg} / \mathrm{mL}$ was the maximum loading concentration for dynamic adsorption of blueberry anthocyanin.

For macroporous resin, ethanol is the preferred desorption agent. It is simple to extract from the solution and recycle and is inexpensive. As shown in Fig. 3d, the desorption rate increased with the ethanol concentration. When the concentration of ethanol was $100 \%$, the desorption rate arrived at the highest, and the difference with that using other ethanol concentrations has statistical significance $(P<0.05)$. Therefore, $100 \%$ ethanol was the optimal condition for the desorption test. Anthocyanins can be desorbed from macroporous resins because of the interaction between the adsorption capacity of macroporous resin and solvent solubility. When the intermolecular forces were recessive, the blueberry anthocyanins were desorbed from the resins into the solvents [21].

The highest elution result was achieved when the elution flow rate was $0.5 \mathrm{~mL} / \mathrm{min}$, as illustrated in Fig. 3e and $\mathrm{f}$. The eluent solvent had sufficient time to interact with anthocyanins on the surface of AB-8 resin. At a flow rate of $0.5 \mathrm{~mL} / \mathrm{min}$, the anthocyanin concentration increased. Approximately $70 \mathrm{~mL}$ of anthocyanin solution was completely poured out of the column. A slow eluent flow increased the time go through the column [9]. In general, raising the elution flow rate has a detrimental effect on the dynamic desorption ability of an eluent solvent on resin. Decreased in elution flow rate extended the working period. The $2.0 \mathrm{~mL} / \mathrm{min}$ elution flow rate was considered suitable due to the short working time and reduced volume consumption. 


\section{Identification of blueberry anthocyanins by UPLC and UPLC-ESI-MS}

Referring to the previous literature data, the individual anthocyanin compounds in blueberries were identified using UPLC-ESI-MS analysis [22-24]. The MS and $\mathrm{MS}^{2}$ spectra of blueberry anthocyanins were shown in Additional file 1 . The results indicated that nine distinct individuals existed in Brightwell blueberry anthocyanins (Table 2). It also revealed that only five of the six most common anthocyanins were in Brightwell blueberry cultivated in Nanjing, namely delphinidin $(m / z 303)$, cyani$\operatorname{din}(m / z 287)$, petunidin $(m / z 317)$, peonidin $(\mathrm{m} / \mathrm{z} 301)$, and malvidin $(\mathrm{m} / \mathrm{z} 331)$. The derivate of pelargonidin was not detected in this study, which was similar to the findings reported by Hutabarat [22].

Peak 1 displayed a spectra pattern $([\mathrm{M}+\mathrm{H}] 465.1 \mathrm{~m} / z)$ with a molecular weight of $303 \mathrm{~m} / z$ plus $162 \mathrm{~m} / z$, indicating that it was provisionally recognized as delphinidin-3-galactoside in the MS analysis. Peak 2 displayed a spectral pattern $([\mathrm{M}+\mathrm{H}] 435.1 \mathrm{~m} / z)$ with a molecular weight of $303 \mathrm{~m} / z$ plus $132 \mathrm{~m} / z$ according to MS analysis. Delphinidin-3-arabinoside was tentatively identified as peak 2. Peaks 3 and 4 had $[\mathrm{M}+\mathrm{H}] 449.2 \mathrm{~m} / z$, indicating that these two components were cyanidin derivatives with molecular weights of $287 \mathrm{~m} / z$ plus $162 \mathrm{~m} / z$. While peak 6 revealed a molecular cation $(\mathrm{M}+\mathrm{H})$ at $610.8 \mathrm{~m} / z$ and a fragment ion at $287.0 \mathrm{~m} / z$. The cyanidin aglycone was separated when the fragment ion at $287.0 \mathrm{~m} / z$ was generated, indicating that the arabinoside $(132 \mathrm{~m} / z)$ was lost. cyanidin-3-arabinoside was tentatively identified as peak 6 . The MS analysis of peak 5 showed a molecular anion at $479.1 \mathrm{~m} / z$ and a significant fragmentation in $\mathrm{MS}^{2}$ at $317 \mathrm{~m} / z$, corresponding to the petunidin aglycone, as a result of galactoside loss $(162 \mathrm{~m} / z)$. Peak 5 was tentatively identified as petunidin -3-galactoside. The mass spectra of peak $7,[\mathrm{M}+\mathrm{H}] 493.1 \mathrm{~m} / z$ with the molecular weight of $331 \mathrm{~m} / \mathrm{z}$ plus $162 \mathrm{~m} / z$, indicated that it was malvidin-3-galactoside. The mass spectra of peak $8,[\mathrm{M}+\mathrm{H}] 463.2 \mathrm{~m} / z$ with the molecular weight of $331 \mathrm{~m} / z$ plus $132 \mathrm{~m} / z$, indicated that it was malvidin3 -arabinoside. In addition, peak 9 had the mass spectra $[\mathrm{M}+\mathrm{H}] 549.1 \mathrm{~m} / z$. The fragment ion $\mathrm{m} / z 303$ [M-16284 ] was obtained by losing one molecule of glucose and one molecule of malonic acid, and $\mathrm{m} / \mathrm{z} 303$ was delphinidin. Therefore, it was inferred that the substance of peak 9 might be delphinidin-3-O-(6"-malonyl glucoside).

Anthocyanins in the rabbiteye blueberry cultivar Delite cultivated in Brazil included the aglycones of delphinidin, cyanidin, delphinidin, and pelargonidin, were slightly different from blueberries cultivated in Nanjing. Petunidin and peonidin derivatives were not detected in these rabbiteye blueberries [24]. These discrepancies likely occur because the composition of anthocyanins in blueberry fruits varies with their original location and preharvest climate factors [22].

\section{Antioxidant properties}

Compound antioxidant activity is also commonly expressed as the percentage of pre-prepared free radical inhibition by antioxidants, and the $\mathrm{EC}_{50}$, the concentration required to achieve a $50 \%$ antioxidant effect, is a commonly used parameter for quantifying and comparing the antioxidant capacities of different compounds [25]. The results of antioxidant properties were presented in Table 3. The effectiveness of antioxidant properties was inversely related to their $\mathrm{EC}_{50}$ values. The $\mathrm{EC}_{50}$ of $\mathrm{BRE}$ for the DPPH assay was estimated to be $0.51 \pm 0.01 \mathrm{mg} /$ $\mathrm{mL}$, which were much lower than those for BCE and BFS $(2.35 \pm 0.13$ and $4.80 \pm 0.08 \mathrm{mg} / \mathrm{mL}$, respectively). The results of the ABTS assay, measured in effective concentration providing $50 \%$ inhibition $\left(\mathrm{EC}_{50}\right)$, were in good agreement with results of the DPPH assay because BRE had the highest antioxidant capacity, followed by BCE and BFS. Significant differences $(P<0.05)$ were observed between BRE and BCE. Regarding the results obtained from the ORAC assay, the total average values were

Table 2 Analysis of anthocyanins in blueberry by LC-MS/MS

\begin{tabular}{lllll}
\hline Peak & TR/min & {$[\mathbf{M}+\mathbf{H}] \mathbf{( m / z )}$} & MS/MS (m/z) & Identified anthocyanins \\
\hline 1 & 3.12 & 465.1 & 303 & Delphinidin-3-galactoside \\
2 & 3.67 & 435.1 & 303 & Delphinidin-3-arabinoside \\
3 & 3.79 & 449.2 & 287 & Cyanidin-3-galactoside \\
4 & 3.82 & 449.2 & 287 & Cyanidin-3-glucoside \\
5 & 4.11 & 479.1 & 317 & Petunidin-3-galactoside \\
6 & 4.39 & 419.1 & 287 & Cyanidin-3-arabinoside \\
7 & 4.99 & 493.1 & 331 & Malvidin-3-galactoside \\
8 & 6.60 & 463.2 & 331 & Malvidin-3-arabinoside \\
9 & 7.28 & 549.1 & 303 & Delphinidin-3-(6" ${ }^{\prime \prime}$-malonyl glucoside) \\
\hline
\end{tabular}


Table 3 Antioxidant activity of BRE, BCE, and BFS

\begin{tabular}{llll}
\hline Samples & DPPH $^{1}$ & ABTS $^{1}$ & ORAC $^{\mathbf{2}}$ \\
\hline BRE & $0.51 \pm 0.01^{\mathrm{c}}$ & $0.32 \pm 0.01^{\mathrm{b}}$ & $0.43 \pm 0.09^{\mathrm{a}}$ \\
BCE & $2.35 \pm 0.13^{\mathrm{b}}$ & $2.59 \pm 0.26^{\mathrm{a}}$ & $0.19 \pm 0.03^{\mathrm{b}}$ \\
BFS & $4.80 \pm 0.08^{\mathrm{a}}$ & $3.04 \pm 0.20^{\mathrm{a}}$ & $0.12 \pm 0.01^{\mathrm{b}}$ \\
\hline
\end{tabular}

${ }^{1}$ Expressed in effective concentration at which $50 \%$ of DPPH or ABTS radicals are scavenged (EC50, $\mathrm{mg} / \mathrm{mL}$ )

${ }^{2}$ Expressed in mmol Trolox/g. Values were presented as the mean $\pm S D(n=3)$

Symbols $\mathrm{a}, \mathrm{b}$, and $\mathrm{c}$ indicate a significant $(P<0.05)$ difference within a column

0.43,0.19, and $0.12 \mathrm{mmol}$ Trolox/g in BRE, BAE, and BFS, respectively. Therefore, BRE presented obvious antioxidant capacity.

\section{Antimicrobial activity of samples}

The crystal violet staining method was used to quantify Listeria monocytogenes biofilms, and the results are shown in Fig. 4. At $2 \mathrm{mg} / \mathrm{mL}$, BRE showed a maximum of $84.64 \pm 0.35 \%$ reduction in biofilm biomass of Listeria monocytogenes. Moreover, the biofilm inhibition rate of Listeria monocytogenes treated with BRE was higher $(P<0.05)$ than the rates in the other groups. Zhang also found that the anthocyanin-rich aqueous extract of purple highland barley bran has a high anthocyanin content and can inhibit the biofilm formation of $P$. aeruginosa PAO1 and S. enterica ATCC10398 [13]. Therefore, high anthocyanin content highly contributes to the antibiofilm activity of BRE.

Markers of antibacterial activities of BRE, BCE, and BFS, including growth inhibition zone, were shown in Table 4. The inhibition zone given by BRE against

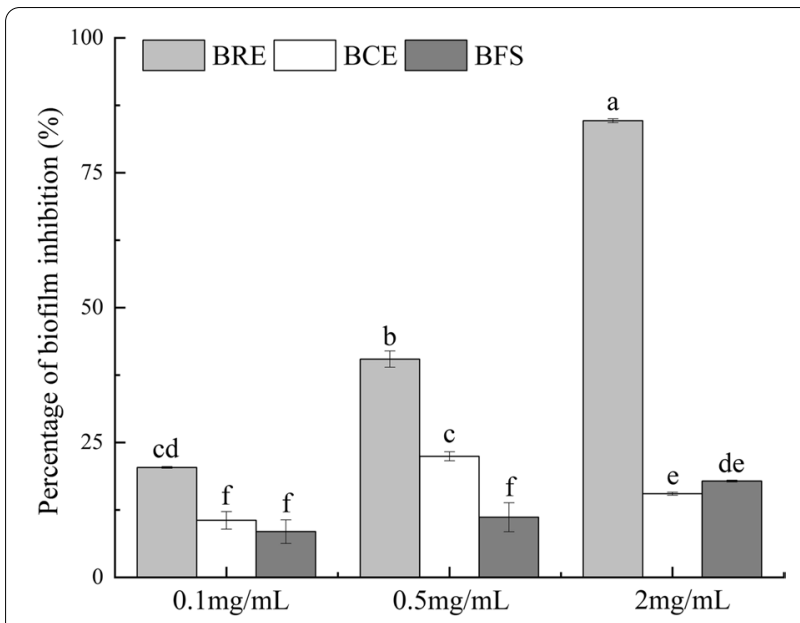

Fig. 4 Effects of samples on biofilm formation in Listeria monocytogenes. Data were represented as the percentage of biofilm inhibition
Table 4 Diameters of inhibition zone (DIZ) of samples against pathogenic bacteria

\begin{tabular}{llll}
\hline Fractions & Salmonella enterica & Escherichia coli & $\begin{array}{l}\text { Listeria } \\
\text { monocytogenes }\end{array}$ \\
\hline BRE & $10.95 \pm 0.64^{\mathrm{a}}$ & $16.04 \pm 0.38^{\mathrm{a}}$ & $\mathrm{NIZ}$ \\
$\mathrm{BCE}$ & $8.80 \pm 0.42^{\mathrm{b}}$ & $12.05 \pm 0.35^{\mathrm{b}}$ & $\mathrm{NIZ}$ \\
$\mathrm{BFS}$ & $8.30 \pm 0.28^{\mathrm{b}}$ & $8.85 \pm 0.50^{\mathrm{c}}$ & $\mathrm{NIZ}$ \\
$\mathrm{CK}$ & $\mathrm{NIZ}$ & $\mathrm{NIZ}$ & $\mathrm{NIZ}$ \\
\hline
\end{tabular}

${ }^{1}$ The means values in each column were compared by ANOVA plus post-hoc Duncan test, and symbols $a$, $b$, and $c$ indicate a significant $(P<0.05)$ difference within a column

NIZ no inhibition zone

Escherichia coli was $16.04 \pm 0.38 \mathrm{~mm}$ (diameter), which was significantly higher than BCE and BFS. The inhibition zones against Salmonella enterica were not as obvious as those against Escherichia coli and Listeria monocytogenes. To Salmonella enterica, the inhibition zone of BRE was larger than BCE and BFS, where no obvious inhibition zones were observed. However, no obvious inhibition zones were observed for Listeria monocytogenes after treatment by all samples. It's possible that this is due to the fact that low-concentration samples cannot produce inhibition zones of Listeria monocytogenes.

\section{Abbreviations}

UPLC-ESI-MS: Ultra-performance liquid chromatography-electrospray ionization interface-mass spectrometer; BFS: The blueberry fruit supernatant; BCE: The blueberry anthocyanin crude extract; BRE: The blueberry anthocyanin rich extract; DPPH: 2,20-Diphenyl-1-picryl-hydrazyl; ABTS: 2,2-Azinobis (3-ethyl benzothiazoline-6-sulfonic acid) diammonium salt; AAPH: 2,2'-Azo-bis(2-amidinopropane) dihydrochloride; Trolox: 6-Hydroxy-2,5,8-tetramethylchroman2-carboxylic acid; RSM: Response surface methodology; DIZ: Diameter of inhibition zone.

\section{Supplementary Information}

The online version contains supplementary material available at https://doi. org/10.1186/s13765-021-00649-8.

\section{Additional file 1: Figure S1. Ion fragments information of Peak 1} (delphinidin-3-galactoside) obtained in UPLC-QTOF-MS. Figure S2. Ion fragments information of Peak 2 (delphinidin-3-arabinoside) obtained in UPLC-QTOF-MS. Figure S3. Ion fragments information of Peak 3 (cyanidin3-galactoside) obtained in UPLC-QTOF-MS. Figure S4. Ion fragments information of Peak 4 (cyanidin-3-glucoside) obtained in UPLC-QTOF-MS. Figure S5. Ion fragments information of Peak 5 (petunidin-3-galactoside) obtained in UPLC-QTOF-MS. Figure S6. lon fragments information of Peak 6 (cyanidin-3-arabinoside) obtained in UPLC-QTOF-MS. Figure S7. Ion fragments information of Peak 7 (malvidin-3-galactoside) obtained in UPLC-QTOF-MS. Figure S8. Ion fragments information of Peak 8 (malvidin3 -arabinoside) obtained in UPLC-QTOF-MS. Figure S9. Ion fragments information of Peak 9 (delphinidin-3-(6"-malonyl glucoside)) obtained in UPLC-QTOF-MS.

Acknowledgements

The authors thank Ning Wang at the Central Laboratory of Jiangsu Academy of Agricultural Sciences for LC-MS/MS technical support. 


\section{Authors' contributions}

Hannan. Liu was responsible for the experimental implementation and the manuscript writing. Han Wu, Ying Wang, Fan Wang and Xiaoli. Liu took part in the data analysis and purchase of reagents and materials. Jianzhong. Zhou was responsible for the experimental design, manuscript writing and financial support. All authors have read and approved the final manuscript.

\section{Funding}

This work was financially supported by the Science and Technology Program of Jiangsu Province [Grant Number BE2020380], and the National Natural Science Foundation of China [Grant Number 31801531].

\section{Availability of data and materials}

Not applicable.

\section{Declarations}

\section{Competing interests}

There are no conflicts of interest to declare.

\section{Author details}

${ }^{1}$ School of Food and Biological Engineering, Jiangsu University, 301 Xuefu Road, Zhenjiang, Jiangsu 212013, P.R. China. ${ }^{2}$ Institute of Agricultural Product Processing, Jiangsu Academy of Agricultural Sciences, Nanjing 210014, P.R. China.

Received: 23 September 2021 Accepted: 28 October 2021 Published online: 05 November 2021

\section{References}

1. Gao Z (2017) Extraction, separation, and purification of blueberry anthocyanin using ethyl alcohol. KUI Chem Ind 66:655-659. https://doi.org/10. 15255/KUI.2017.041

2. Hosseinian FS, Beta T (2007) Saskatoon and wild blueberries have higher anthocyanin contents than other manitoba berries. J Agr Food Chem 55:10832-10838. https://doi.org/10.1021/jf072529m

3. Ma L, Sun Z, Zeng Y, Luo M, Yang J (2018) Molecular mechanism and health role of functional ingredients in blueberry for chronic disease in human beings. Int J Mol Sci. https://doi.org/10.3390/ijms19092785

4. Castañeda A, Pacheco HL, Páez E, Rodriguez J, Galán-Vidal C (2009) Chemical studies of anthocyanins: a review. Food Chem 113:859-871. https://doi.org/10.1016/j.foodchem.2008.09.001

5. Diaconeasa Z, Leopold L, Rugină D, Ayvaz H, Socaciu C (2015) Antiproliferative and antioxidant properties of anthocyanin rich extracts from blueberry and blackcurrant Juice. Int J Mol Sci 16:2352-2365. https://doi. org/10.3390/ijms16022352

6. Jing P, Ruan S-Y, Dong Y, Zhang X-G, Kan J-Q, Slavin M (2011) Optimization of purification conditions of radish (Raphanus sativus L.) anthocyanin-rich extracts using chitosan. LWT Food Sci Technol 44:2097-2103. https://doi. org/10.1016/j.lwt.2011.06.003

7. Sun XH, Zhou TT, Wei CH, Lan WQ, Zhao Y, Pan YJ, Wu VH (2018) Antibacterial effect and mechanism of anthocyanin rich Chinese wild blueberry extract on various foodborne pathogens. Food Control. https://doi.org/ 10.1016/j.foodcont.2018.07.012

8. Kong S, Lee J (2010) Antioxidants in milling fractions of black rice cultivars. Food Chem 120:278-281. https://doi.org/10.1016/j.foodchem.2009. 09.089

9. Yang Y, Yuan X, Xu Y, Yu Z (2015) Purification of anthocyanins from extracts of red raspberry using macroporous resin. Int J Food Prop 18:1046-1058. https://doi.org/10.1080/10942912.2013.862632

10. Zhou L, Xie M, Yang F, Liu J (2019) Antioxidant activity of high purity blueberry anthocyanins and the effects on human intestinal microbiota. LWT Food Sci Technol 117:108621-108621. https://doi.org/10.1016/j.lwt. 2019.108621
11. Cerezo AB, Ctunescu GM, González M, Hornedo-Ortega R, Troncoso A (2020) Anthocyanins in blueberries grown in hot climate exert strong antioxidant activity and may be effective against urinary tract bacteria. Antioxidants. https://doi.org/10.3390/antiox9060478

12. Luo T, Chen S, Zhang H, Jia S, Wang J (2020) Phytochemical composition and potential biological activities assessment of raspberry leaf extracts from nine different raspberry species and raspberry leaf tea. J Berry Res 10:1-15. https://doi.org/10.3233/JBR-190474

13. Zhang YZ, Lin YF, Huang L, Tekliye M, Rasheed HA, Dong MS (2020) Composition, antioxidant, and anti-biofilm activity of anthocyanin-rich aqueous extract from purple highland barley bran. LWT Food Sci Technol. https://doi.org/10.1016/j.lwt.2020.109181

14. Gan RY, Kong KW, Li HB, Wu K, Ge YY, Chan CL, Harold C (2018) Separation, identification, and bioactivities of the main gallotannins of red sword bean (Canavalia gladiata) coats. Front in Chem. https://doi.org/10.3389/ fchem.2018.00039

15. Kang YJ, Jung SW, Lee SJ (2014) An optimal extraction solvent and purification adsorbent to produce anthocyanins from black rice (Oryza sativa cv. Heugjinjubyeo). Food Sci Biotechnol 23:97-106. https://doi.org/10. 1007/s10068-014-0013-8

16. Khoo HE, Azlan A, Tang S, Lim S (2017) Anthocyanidins and anthocyanins: colored pigments as food, pharmaceutical ingredients, and the potential health benefits. Food Nutr Res 61:1361779. https://doi.org/10.1080/16546 628.2017 .1361779

17. Torskangerpoll K, Andersen $\varnothing$ (2005) Colour stability of anthocyanins in aqueous solutions at various pH values. Food Chem 89:427-440. https:// doi.org/10.1016/j.foodchem.2004.03.002

18. Dong J, Liu Y, Liang Z, Wang W (2009) Investigation on ultrasoundassisted extraction of salvianolic acid B from salvia miltiorrhiza root. Ultrason Sonochem 17:61-65. https://doi.org/10.1016/j.ultsonch.2009.05. 006

19. Zou T-B, Wang M, Gan R-Y, Ling W-H (2011) Optimization of ultrasoundassisted extraction of anthocyanins from mulberry, using response surface methodology. Int J Mol Sci 12:3006-3017. https://doi.org/10. 3390/ijms12053006

20. Zhao Z, Wu M, Zhan Y, Zhan K, Chang X, Yang H, Li Z (2017) Characterization and purification of anthocyanins from black peanut (Arachis hypogaea L.) skin by combined column chromatography. J Chromatogr A 1519:74-82. https://doi.org/10.1016/j.chroma.2017.08.078

21. Xue H, Shen L, Wang X, Liu C, Liu C, Liu H, Zheng X (2019) Isolation and purification of anthocyanin from blueberry using macroporous resin combined sephadex LH-20 techniques. Food Sci Technol Res 25:29-38. https://doi.org/10.3136/fstr.25.29

22. Hutabarat RP, Xiao YD, Wu H, Wang J, Li DJ, Huang WY (2019) Identification of anthocyanins and optimization of their extraction from rabbiteye blueberry fruits in Nanjing. J Food Quality 2019:1-10. https://doi.org/10. $1155 / 2019 / 6806790$

23. Reque P, Steffens R, Martins da Silva A, Jablonski A, Flôres S, Rios A, Jong E (2014) Characterization of blueberry fruits (Vaccinium spp.) and derived products. Food Sci and Tech 34:773-779. https://doi.org/10.1590/1678$457 X .6470$

24. Wu X, Prior RL (2005) Systematic identification and characterization of anthocyanins by HPLC-ESI-MS/MS in Common Foods in the United States: fruits and berries. J Agr Food Chem 53:2589-2599. https://doi.org/ 10.1021/jf048068b

25. Xiao Y, Rui X, Xing G, Wu H, Li W, Chen X (2015) Solid state fermentation with Cordyceps militaris SN-18 enhanced antioxidant capacity and DNA damage protective effect of oats (Avena sativa L.). J Funct Foods 16:58-73. https://doi.org/10.1016/j.jff.2015.04.032

\section{Publisher's Note}

Springer Nature remains neutral with regard to jurisdictional claims in published maps and institutional affiliations. 